\title{
Las ediciones latinas de las obras de Aristóteles en la Edad Media, en relación con los comentarios de Tomás de Aquino
}

Oscar Jiménez Universidad Panamericana

The author shows briefly: a) recognized critical editions of Aristotle's Complete Works; b) the twelve Commentaries of Saint Thomas Aquinas and the translations in which he had based for his Commentaries; c) the problems of dating the translation of Book $\mathrm{XI}($ Kappa) of Aristotle's Metaphysics, and some consequences in relation with the respective Commentary of Aquinas; d) Some pseudo-Aristotelian works that were mentioned by Thomas Aquinas, but not commented by him, in the Commentary on De Sensu et Sensato.

En estas notas estudiamos algunas características de las ediciones de las obras de Aristóteles traducidas al latín sobre las que trabajaban los maestros en la Edad Media (s. XIII principalmente), y, en específico, Tomás de Aquino. En los comentarios de Aquino a los textos aristotélicos encontramos afirmaciones como: "aliam litteram habet...", "sed secundum litteram aliam...", etc., expresiones que dejan ver que tenía por lo menos otra traducción de las obras de

${ }^{1}$ En nueve de doce comentarios del Aqu

incluimos De causis, prostételes (o trece, si aparecen estas causis, pseudo-aristotélico pero que también explicó Santo Tomás), confrontación expresiones "secundum aliam litteram", que indican una comentarios con otra versión latina del texto griego. En efecto, esos nueve caelo et mundes Aquino son sobre las siguientes obras aristotélicas: Physica, De caelo et mundo, De generatione et corruptione, De anima, De memoria et Metaphysicentia, De Sensu et Sensato, Peri Hermeneias, Ethica Nichomachea y doce obras de ano obras de Aristóteles que comentó Tomás de Aquino. Como apéndice (No. 6), anotamos todos los lugares donde Aquino afirma "secundum aliam litteram" en sus comentarios al corpus aristotélico. 
Aristóteles al comentarlas. ${ }^{2}$ Además, Tomás menciona a lo largo de sus comentarios algunos libros pseudo-aristotélicos que también serán objeto de nuestra indagación.

Los comentarios de Santo Tomás al corpus de Aristóteles son parte importante de la doctrina del Aquinate y no sólo un apéndice de su teología, lo cual se muestra en sus obras teológicas principales en donde supone tesis filosóficas aristotélicas. Las doctrinas de la materia y la forma, del acto y la potencia, de la substancia y los accidentes, etc., son todas ellas utilizadas para responder a graves problemas teológicos. No es, pues, superficial abordar el estudio de los comentarios de Tomás de Aquino a las obras de Aristóteles.

Como marco general, veremos cuáles son algunas ediciones críticas de las obras del Estagirita, así como datos generales de los indices de voces aristotélicas. Posteriormente, mostraremos sobre qué textos estudiaba Aquino las obras de Aristóteles "latino". También haremos algunas anotaciones particulares sobre la Metaphysica de Aristóteles y los códices respectivos que tenía Santo Tomás a la vista. Finalmente, llevaremos a cabo un recuento de las obras aristotélicas que el Aquinate menciona en su comentario al libro De Sensu et Sensato, puesto que es el lugar donde reseña a la vez más obras del Estagirita en sus comentarios.

\section{1) Ediciones críticas de las obras completas de Aristóteles y apoyo bibliográfico aristotélico}

Actualmente contamos con las obras críticas de Aristóteles en las versiones que durante el siglo XIX se llevaron a cabo, principal aunque no únicamente, por editores alemanes. Para apreciar los títulos de esas ediciones, podemos remitir al lector al Prólogo que realiza Valentín García Yebra, traductor al castellano de la Metaphysica de Aristóteles, para introducir su versión trilingüe. ${ }^{3}$

${ }^{2}$ Hemos colaborado en un trabajo al respecto y sobre el cual volveremos más adelante: Jorge MORAN: “Anicio Manlio Severino Boecio: notas a una traducción inédita a la Metafísica de Aristóteles", Tópicos 18 (2000), pp. 143-166.

${ }^{3}$ ARISTÓTELES: Metaphysica, Madrid: Gredos,1991. Versión de Valentín García Yebra, Prólogo, pp. XI-LIII a) Una de las principales es la edición de Bekker: "Aristotelis OPERA", ex recensione IMMANUELIS BEKKERI, edidit Academia Regia Borussica, Berlin, 1831-1870. 5 vols. El texto griego de Aristóteles, como dice García Yebra (ARISTOTELES Graece), ocupa los volúmenes I y II, y la Metaphysica, las páginas 980 a 1093 del vol. II. ${ }^{4}$

b) Otra edición importante de las obras completas de Aristóteles fue llevada a cabo por August Firmin Didot, también en el siglo XIX. El título es Aristotelis Opera Omnia graece et latine cum indice nominum et rerum absolutissimo, 5 vols. Instituto Franciae Typographo, Paris, 1874-1878. La edición de Didot se divide en dos columnas (texto griego y latino respectivamente), siguiendo la numeración línea por línea de Bekker en el texto griego. Esta versión - que no es sino revisión de otras anteriores, según dice Didot en el Prólogo de su libro—S está a cargo de Fridericum Dübnerum.

c) El Index Aristotelicus de Bonitz data del año $1870 .^{6}$ Como se sabe, Bonitz utiliza la numeración de Bekker para citar las voces aristotélicas griegas. A su vez, Bonitz conoce la edición de Didot de 1848 pues está citada al inicio de su libro, siendo su propia obra un instrumento de trabajo vigente para el estudioso de Aristóteles.

\footnotetext{
${ }^{4}$ La edición de Bekker está ordenada de manera tal que aún actualmente se utiliza su numeración como 'canónica'. Divide el texto griego de Aristóteles en páginas (numeración arábiga), las cuales tienen dos columnas (cuya nomenclatura es ' $a$ ' y 'b') y cada columna tiene un promedio de 35 líneas. Asi, la primera obra ordenada por Bekker, las Categoriae, comienza en la página 1, columna a, línea 1. Por ello, una obra de Aristóteles en la edición de Bekker se cita comúnmente: Categoriae la 1. Es decir, primera página, columna a, línea 1. La Metaphysica, por ejemplo, comienza en la página 980, columna a, número 21, y se cita: Metaphysica 980a 21 . En la edición O. Gigon de la obra de Bekker de 1960 (Aristotelis Opera ex recensione Immanuelis Bekkeri, edidit Academia Regia Borussica, Editio Altera, Addendis instruxit fragmentorun collectionem retractavit Olof Gigon, Berolini Apud W. De Gruyter et socios, MCMLX), el lector puede encontrar un listado de las ediciones que se han hecho de una o varias obras de Aristóteles entre 1834 y 1959 , así como de los códices respectivos utilizados. Cf. Aristotelis Opera..., pp. XI-XXI. ${ }_{5}$ ARISTóteles: Opera Omnia..., Praefatio, p. Il y VI.

${ }^{6}$ Hermann BonITZ: Index Aristotelicus, Graz: Akademische Druck-U. Verlagsansalt,
} 
d) El volumen $\mathrm{V}$ de la edición de Didot es un índice de las voces aristotélicas, y fue publicado tres años después que el Index de Bonitz. La diferencia es que el índice de la edición Didot no es de términos griegos - como el de Bonitz-, sino de voces latinas, citando los IV volúmenes previos. El Volumen $\mathrm{V}$ de la edición Didot se titula: Aristotelis Opera Omnia: Index Nominum et Rerum Absolutissimus, fechado en 1873. En el prólogo de su edición, el compilador francés cita al index Bonitzianus.?

e) El apoyo lexicográfico que proporciona el CD-ROM Thesaurus Linguae Graecae es imprescindible para localizar un término específico en todo el corpus de los autores griegos, desde el siglo VIII a.C. hasta 1453 d.C. Tal labor de recopilación de textos es insoslayable para la búsqueda de obras completas así como de términos específicos de un autor griego o helénico, sea poeta, filósofo, teólogo, cómico, etc.

The TLG Digital Library contains virtually all ancient Greek texts surviving from the period between Homer (8th century B.C.) and A.D. 600, and a large number of texts deriving from the period between A.D. 600 and the fall of Byzantium in 1453. Byzantine historiographical and lexicographical works have already been digitized and are included in this disk. $^{8}$

La colección contiene cerca de 11,000 trabajos de casi 3,000 autores y un aproximado de 800 millones de palabras.

\footnotetext{
7 Aristotelis Opera Omnia: Index Nominum et Rerum Absolutissimus. Volumen Quintum Continens Indicem Nominum et Rerum, Parisiis: Editore Ambrosio Firmin Didot, 1873. Praefatio Aem. Heitz. p. VI.

${ }^{8}$ Thesaurus Linguae Graecae, University of California, 1999, Irvine. 3450, Berkeley Place, Irvine, CA. Proyect Director, Maria C. Pantella. Véase en internet: http://www.tlg.uci.edu/ tlg.
}

\section{2) Versión latina de las obras de Aristóteles: "Aristoteles Latinus"}

Antes de abordar el estudio de las traducciones de la obra aristotélica que poseían los maestros del siglo XIII, conviene anotar la Edición: Aristoteles Latinus. ${ }^{9}$ Es la edición de las obras completas de Aristóteles vertida al latín, en la cual se encuentran recogidas traducciones diversas a una misma obra. Por ejemplo, en esta edición se encuentran las versiones llamadas por la crítica: Vetustissima, Media, Nova, etc., a las que volveremos más adelante. El estudioso de los comentarios de Tomás de Aquino en relación con Aristóteles puede confrontar las versiones que el Aquinate utilizaba para redactar su texto. En el caso específico de la Metaphysica, hasta donde sabemos, todavía no hay una edición en la cual veamos qué traducción seguía Aquino en determinados párrafos, o en cuáles cambiaba de texto latino y cuál era el que citaba de modo específico. La Edición Leonina del Comentario a la Metaphysica podría ser esa edición crítica monumental, aunque no ha sido publicada aún.

Una de las principales autoridades para hablar de los códices latinos que tenían los pensadores medievales a la mano es George Lacombe. En su obra homónima, complementaria a la edición que acabamos de citar: Aristoteles Latinus, ${ }^{10}$ Lacombe recopila los códices latinos de los textos aristotélicos y pseudo-aristotélicos en manos de los escritores medievales, traducidos ya del griego ya del árabe. Como se dice en el prólogo, en la Unión Académique Internationale intentaron tal obra: "cuius operis primum locum Aristotelem Latinum, id est sive ex graeco sive ex arabico mediae aetatis latinas librorum aristotelicorum vel Aristoteli tributorum

\footnotetext{
9 Aristoteles Latinus, Bruges-Paris: Desclée de Brouwer,1961, Edidit Laurentius Minio-Paluello, Corpus Philosophorum Medii Aevi Academiarum Consorciatarum Auspiciis et Consilio Editum, Union Académique Internationale.

${ }^{10}$ Aristoteles Latinus, Codices descripsit Greorgius LACOMBE, in societate operis adsumptis, A. Birkenmajer, M. Dulong, Aet. Franceschini, Roma: La Librería dello Stato, 1939. Corpus Philosophorum Medii Aevi, Academiarum Consorciatarum Auspiciis et Consilio Editum, Union Académique Internationale. Citaremos ect?
} 
translaticnes ad criticae artis rationem editas, obtinere voluerunt". ${ }^{11}$ Como se ve, es el trabajo paralelo a la publicación Aristoteles Latinus, en cuanto que aquella edición es la labor final, y esta obra de Lacombe es la muestra de todos los códices en los cuales se trabajó para presentar dicha edición crítica.

La división del catálogo que se ofrece en el texto de Lacombe es la siguiente:

\section{Bibliografia selecta de las obras de Aristóteles.}

II. Índice de los tratados aristotélicos que se conocían en la Edad Media, según traducciones que aún existen.

III. Datos de las versiones y recensiones de cada uno de esos tratados, según este orden: a) Libros lógicos; b) Libros naturales; c) Libros metafísicos; d) Libros morales; e) Libros de los animales; f) Obras pseudo-aristotélicas; g) Comentadores griegos de Aristóteles; y h) Comentarios de Averroes sobre Aristóteles. ${ }^{12}$

IV. Traducción de todos los fragmentos que hay.

\section{Catálogo de los códices. ${ }^{13}$}

En tal labor de recopilación nos hemos basado para exponer a continuación los posibles traductores de las 12 obras de Aristóteles que comentó Santo Tomás, así como algunos textos pseudoaristotélicos que solamente menciona, aunque sí comentó su maestro Alberto Magno.

11 G. LACOMBE: Aristoteles Latinus..., Prooemium. p. 5.

12 Los comentarios de Averroes se encuentran clasificados en el Volumen I de la obra. Ver, I, 100-111. La clasificación de las obras de Averroes sobre la doctrina aristotélica se dividen en cuatro géneros: a) Comentarios magnos (commentaric magna); b) Comentarios medios (commentaria media); c) Compendios (compendia), y d) Cuestiones (quaestiones). Las cuestiones no corresponden propiamente a esa recopilación de Lacombe, razón por la cual no se citan en el libro. Cf. G. LACOMBE: Aristoteles Latinus..., I, 100.

${ }^{13}$ G. LACOMBE: Aristoteles Latinus..., p. 8. a) Traducciones disponibles en el siglo XIII de las obras de Aristóteles

Los pensadores medievales contaban con diversas traducciones de libros aristotélicos que podían confrontar para acercarse más al sentido de la 'littera Aristotelis'. Según De Wulf, ${ }^{14}$ a partir de la reconquista cristiana de Toledo, en 1085, comienza una labor de difusión de la ciencia sin parangón en todo Occidente. El arzobispo Raimundo (1126-1152) reunió un colegio de traductores, de entre los cuales los principales son: el segoviano Domingo Gundisalvo (Gundissalinus), traductor de Avicena; su colaborador judio Juan Avendehut (Iohannes Hispanus, Hispaliensis); ${ }^{15}$ el lombardo Gerardo de Cremona, ${ }^{16}$ muerto en 1187; así como Alfredo de Sareshel (Alfredus Anglicus). ${ }^{17}$ Otro traductor renombrado del siglo XII es Enrique Aristipo, quien tradujo el libro IV de los Meteorologica de Aristóteles.

Más adelante, durante el mismo siglo en que vivió Tomás de Aquino, s. XIII, Miguel Escoto, primero en Toledo y después en Palermo con Federico II, se constituyó como uno de los principales traductores -y por ende, difusores- de la doctrina aristotélica entre los

14 DE Wulf: Historia de la Filosofia Medieval, Traducción de Jesús Toral Moreno, México: Jus 1945, Tomo I. p. 64.

15 Tradujo escritos astronómicos de Albategni (Al-Battani), Alchabitius (AlKabi'si), Alfraganus (Al-Fergani), Albumasar (Abu -Battani), Alchabitius (Altraducidas: De Intellectu, de Al Kindi, y el tratado que caso por aristicicas differentia inter animam et spiritum, fel filón otras obras. De Avicen curación curación (llamado erróneamente por los latinos: Sufficientia).

16 Tradujo Analytica Posteriora y el comentario de Temistio, así como la Physica (De naturali auditu) del árabe al latín; De Caelo et Mundo, De Generatione y los aristerimeros libros de los Meteorologica. A su vez, tradujo textos pseudoaristotélicos tales como el primer tratado y el fin del segundo tratado: De causis proprietarum et elementorum, asi como el Liber de Causis o Liber expositione bonitatis purae.

${ }^{17}$ Tradujo De vegetabilibus falsamente atribuido a Aristóteles, que es, según de Wulf, De plantis de Nicolás de Damasco. A su vez, tradujo el pseudo-aristotélico De Mineralibus o De Congelatis (De congelatione et conglutinatione lapidum, es un escrito aviceniano en realidad). A este libronglinatione lapidum, que Mineralibus en su 'Propemium' el cnmentarin libro Santo Tomás lo llama De 
latinos medievales. Es conocido principalmente por traducir los comentarios de Averroes a las obras aristotélicas, hacia 1220 ó $1230 .{ }^{18}$ Uno de los más ilustres fue Guillermo de Moerbeke, al cual volveremos más adelante, aunque su impronta fue mucho más tardía, hablando del siglo XIII.

Lacombe en el segundo tomo de su obra nos ofrece la relación completa y resumida de las obras que los latinos poseían de Ariștóteles, así como los traductores de las mismas. Algunos de los libros fueron traducidos desde el griego, otros desde el árabe, o, incluso, del hebreo. Remitimos al lector a ese tomo II, en el Supplementum. ${ }^{19}$ Resumiremos aquí, tomando el índice de Lacombe, las traducciones de los textos de Aristóteles en los siglos XII-XIV. Las 13 obras que ponemos son las que Santo Tomás de Aquino comentó ya parcial ya totalmente, aunque el índice de Lacombe se extiende a todo el corpus aristotelicum. Con este listado no decimos que Aquino tuvo en sus manos todas estas traducciones, sino que en algunos comentarios se nota que por lo menos tuvo dos diversas a la mano:

\section{i) De Interpretatione: ${ }^{20}$}

a) Traducción de Boecio;

b)Traducción de Guillermo de Moerbeke.

\section{ii) Analytica Posteriora: ${ }^{21}$}

a) Traducción de Jacobo Veneto;

\footnotetext{
18 James WeISHEIPL: Tomás de Aquino, Vida y obras, Versión de Frank Hevia, Pamplona: EUNSA 1994, p. 36

${ }^{19}$ G. LACOMBE: Aristoteles Latinus..., Vol. II, Supplementum, pp. 783-786.

${ }^{20}$ Tomaremos de Weisheipl los nombres de los respectivos comentarios de Santo Tomás y los datos generales de los mismos. El comentario de Tomás de Aquino a Peri Hermeneias es llamado: Sententiae super Peri Hermeneias. Incompleto; termina en II, 14, 19b 26 - II, Lect. XIV-. (Paris, 1270-1271). Para los nombres de los comentarios de Aquino ver: J. WEISHEIPL: Tomás de Aquino..., pp. 428 y ss.

${ }^{21}$ Comentario de Tomás de AQUINO: Sententia super Posteriora Analytica. Dos libros (Paris, 1269-1272).
}

b) Revisión de Guillermo de M:

c) Traducción Anónima;

d) Traducción de Gerardo de Cremona.

iii) Physica: ${ }^{22}$

a) Traducción de Jacobo V. Nueva revisión y Prólogo ( De intelligentia);

b) Traducción Vaticana (libros I-III);

c)Traducción de Gerardo C.;

d) Traducción de Miguel Escoto (?).

iv) De Caelo: ${ }^{23}$

a) Traducción de Gerardo C.;

b)Traducción de Miguel E. (?);

c) Traducción de Roberto Grosseteste (Libro II, y fragmentos del I y III);

d) Traducción de Guillermo M.

v) De Generatione et Corruptione: ${ }^{24}$

a) Traducción anónima y revisión nueva;

b) Traducción de Gerardo C.

\section{vi) Meteorologica: ${ }^{25}$}

a) Traducción de Gerardo C. (libros I-IV, i);

b) Traducción de Enrique Aristipo (libro IV);

\footnotetext{
${ }^{22}$ Comentario de Tomás de AQUINO: Sententia super Physicam. Ocho libros (París, 1270-71).

${ }^{23}$ Comentario de Tomás de AQuino: Sententia super de Caelo et Mundo. 24 Incompleto; termina en III, 3, 302b 9-III, Lect. VIII-(Nápoles, 1272-1273).

${ }^{24}$ Comentario de Tomás de AQUINO: Sententia super libros De Generatione et Corruptione. Incompleto; termina en I, 5, 322a $33-1$, Lect. XVII- (Nápoles,
1272-1273). ${ }^{25}$ Comentario de Tomás de AQUINO: Sententia super Meteora. Incompleto; termina
en II, 8, 369a 9 (Paris o Nánoles 1260.1272 )
} 
c) Traducción de Guillermo M.;

d) Apéndice A: De causis proprietatum elementorum. Traducción de Gerardo C.;

e) Apéndice B: De minerabilibus. Traducción de Alfredo de Sareshel.

vii) De Anima: ${ }^{26}$

a) Traducción de Jacobo V. y revisión nueva;

b) Traducción de M. Escoto (?).

viii) De Sensu et Sensato:27

Traducción anónima y revisión nueva.

ix) De Memoria et Reminiscentia: ${ }^{28}$

Traducción de Jacobo V., y revisión nueva.

x) Metaphysica: ${ }^{29}$

a) Traducción de Jacobo V. ("vetustissima": libros I-IV, iv);

b) Traducción anónima ("media": libros I-X, XII-XIV);

${ }^{26}$ Comentario de Tomás de AQUINO: Sententia super De Anima. Tres libros. (Italia, 1267-1269). Según Weisheipl (Cf. J. WeISHEIPL: Tomás de Aquino..., p. 431), quien sigue los catálogos más tempranos del texto, el comentario al libro I es una reportatio de Reginaldo de Piperno, mientras que el comentario de los libros II-III es una expositio.

27 Comentario de Tomás de AQUINO: Sententia de Sensu et Sensato. Fecha desconocida.

${ }^{28}$ Comentario de Tomás de AQUINO: Sententia de Memoria et Reminiscentia. Fecha desconocida.

29 Comentario de Tomás de AQUINO: Sententia super Metaphysicam. Doce libros. (Paris, Nápoles [?], 1269-1272). La versión en la que se basa no siempre es la de Moerbeke. En los libros II-III se refiere constantemente al XII (Lambda) como el libro XI, pero - según- la redacción parece posterior a su exposición del libro V, Lect. VII y hasta el libro VII, lect. XVI. Asi, los libros I y IV también serian tempranos, pero a partir del libro VII, lect. XVII y hasta el final del libro XII, serían posteriores, pues cita el libro Lambda como el XII. J. WeishEIPL: Tomás de Aquino..., pp. 432-433. c) Revisión y traducción de Guillermo M. ("nova translationis");

d) Traducción de Miguel E. (?) ("nova": libros II, I. v-x, III-

X, XII);

e) Apéndice: De principiis (=Metaphysica Theophrasti). Traducción de Bartolomeo de Messana.

xi) Ethica Nichomachea: ${ }^{30}$

a) Traducción anónima ("Ethica nova"= libro I; "Ethica vetus": libros II-III; "Ethica Borghesiana": fragmentos de los libros VII y VIII);

b) Revisión (libros I-III) y traducción (IV-X) de Roberto Grosseteste, y nueva revisión (Guillermo de M. [?]).

xii) Politica: ${ }^{31}$

a) Traducción anónima (libros I-III);

b) Revisión (libros I-II) y traducción (libros III-VIII) de Guillermo M.

xiii) De Causis (De expositione bonitate purae). ${ }^{32}$

${ }^{30}$ Comentario de Tomás de AQUINO: Sententia libri Ethicorum. Diez libros. (París, 1271).

${ }^{31}$ Comentario de Tomás de AQUINO: Sententia libri Politicorum. Incompleto; termina en III, 6, 1280a 6 - III, Lect. VI-(Probablemente Paris, 1269-1272).

32 Comentario de Tomás de AQUnNO: Expositio super librum De Causis. (París, 1271-1272). Incluimos De Causis a pesar de ser pseudo-aristotélico, pues Tomás de Aquino también lo comentó. Según García Marqués y José Antonio Fernández, en el comentario al I libro de las Sententiae se designa como "Philosophus" 8 veces de 20 que aparece el autor del Liber. En el comentario al libro II (d. 18, q. 2, a. 2, ad lum) se lee: "Ad primum ergo dicendum quod in hac materia auctoritates lib. De causis recipiendae non sunt; quia ille qui librum illum composuit, huius erroris auctor fuisse invenitur". A partir de ese momento Tomás deja de mencionar al autor del Liber como "Philosophus". Como vemos, no era desconocido a Santo Tomás que el autor del libro De Causis no había sido Aristóteles. La cuestión es saber cuándo se dio cuenta de tal atribución. Cf. Santo Tomás de Aquino, Exposición al 'De Trinitate' de Boecio. Introducción, traducción y notas de Alfonso García Marqués y José Antonio Fernández, Pamplona: EUNSA, 1986, pp. 25-26. 
Traducción de Gerardo C.

La bibliografia de las traducciones, así como de los posibles traductores, está tomada en su totalidad de Lacombe. ${ }^{33}$ Para ver la descripción detallada de estas versiones hay que remitirse al volumen I. ${ }^{34}$ Ahora bien, G. Lacombe en el prólogo de su obra resume una idea que ha desarrollado a lo largo del mismo - de la cual se hace eco García Yebra en su traducción a la Metaphysica. ${ }^{35}$ Casi traduciendo el texto latino, la idea es la siguiente: las obras de Aristóteles que Guillermo de Moerbeke tradujo al latín, ya existían traducidas anteriormente (lo cual acabamos de citar). Dicho traductor corrigió en parte De Caelo et Mundo, libros I-II; Politica, libros I-II; De Partibus Animalium; y en parte no lo hizo por alguna causa desconocida (caso de la Rhetorica, pues no se basó en versiones anteriores). ${ }^{36}$ Francisco Pelster S.J., detectó a partir de la Metaphysica Mediae Translationis, que Guillermo sólo tradujo un libro de la Metaphysica de Aristóteles, y los otros sólo los corrigió. ${ }^{37}$

Resumamos en dos puntos lo que acabamos de citar:

i) En primer lugar, las anotaciones que están en las páginas anteriores con el título de "traducción de Moerbeke", deben entenderse tal y como Lacombe (y Weisheipl que lo sigue) lo

${ }^{33}$ G. LACOMBE: Aristoteles Latinus..., II, pp. 783-786 (Para el libro De Causis, ver I, 94). Cf. también el suplemento que se encuentra enseguida de esta compilación, e cual nosotros hemos resumido para centrarnos en Tomás.

${ }^{34}$ G. LACOMBE: Aristoteles Latinus..., I, 43-95. Recuérdese que nosotros sólo mencionamos las obras de Aristóteles que comentó Santo Tomás. También en ese mismo tomo se encuentran los códices de los comentaristas griegos de Aristó ese vertidos al latín asi como los comentarios de Averroes. G. LACOMBE: Aristoteles Latinus..., 1, 95-111.

35 ARISTÓTELES: Metaphysica, Versión de Valentin García Yebra, Madrid: Gredos 1991, Prólogo, p. XIX.

${ }^{36}$ G. LACOMBE: Aristoteles Latinus..., I, 77.

37 F. PELSTER, S.J.: Die griechisch-lateinischen Metaphysikübersetzungen des Mittelalters, en "Beitrage zur Gesch. der Philos. des Mittelalters", Supplementband II, Münster i. W., 1923, pp. 89-118. En G. LACOMBE: Aristoteles Latinus Praefatio, p. 19. entiende: Guillermo de Moerbeke sólo tradujo a partir del griego y sin antecedente alguno de traducción latina, el libro XI de la Metaphysica, aunque revisó concienzudamente -cotejando con el original $\_{ }^{38}$ todas las obras en donde él aparece como traductor en la lista de Lacombe ya citada. ${ }^{39}$

38 J. WeISHEIPL: Tomás de Aquino..., p. 275. "Desafortunadamente, no conocemos las fechas en que Moerbeke revisó las traducciones latinas de Aristóteles. Guillermo estaba más interesado en hacer nuevas traducciones que en revisar las antiguas. Sin embargo, revisó la mayor parte de las traducciones existentes de Aristóteles, cotejándolas cuidadosamente con el texto griego".

39 Afirma de De Wulff al respecto: "Acerca de la labor de traductor y revisor, Birkenmajer advierte que Guillermo trasladó al latín, para uso de sus amigos, en ocasiones no tratados enteros sino partes de obras, y que otras veces, en su versión de los comentarios, tradujo de diversa manera pasajes de Aristóteles que ya habia traducido con los escritos de los que forman parte. Así vemos que es preciso ser prudentes y reservados para atribuir o no a Guillermo tal o cual versión, en ausencia de testimonios formales". DE WULFF: Historia de la filosofia medieval..., II, 43. Respecto de la obra de Guillermo, a la categoria de traducción pertenecen: Politica (ignorada por los árabes, dice de Wulff [DE WULFF: Historia de la filosofia ..., II, p. 43], y por los latinos, excepto los dos primeros libros que comenta Alberto Magno); Rhetorica; escritos zoológicos de Aristóteles (De Partibus Animalium, 1260); libros I-III de Meteorologica; y libro XI (Kappa) de la Metaphysica. Posiblemente también tradujo: Rhetorica Aristotelis ad Alexandrum y la Oeconomica. A la categoría de revisión de los trabajos ya existentes se comprenden los restantes libros de la Metaphysica, sirviéndose de la Metaphysica Media y la Metaphysica Vetus; la revisión de una gran parte de los libri naturales, y posiblemente de la Ethica Nichomachea (DE WULFF: Historia de la filosofia..., l, p. 45). A esa lista se añaden versiones de diferentes comentarios: Alejandro de Afrodisia sobre los Meteorologica (1260, Nicea) y sobre De Sensu et Sensibili. Además, la traducción de los comentarios de Simplicio a las Categoriae (1266) y De Caelo et Mundo (1271, Viterbo); comentario de Juan Gramático, Alejandrino o Filopón, sobre la parte relativa a la inteligencia, del De Anima, III, 4-8 y principio del 9 (429a 10432a 17) [1268, Viterbo]. Traducción del comentario de Temistio al De Anima, y quizá del comentario de Amonio al Peri Hermeneias. También se encuentra la traducción a la Elementatio Theologica de Proclo (Viterbo, 1268); y tres opúsculos: De providentia et fato et eo quod in nobis, De decem dubitationibus circa providentiam, De malorum subsistentia (Corinto, 1280). A su vez, se le atribuye la versión latina de los Pneumatica de Herón, así como el comentario de Simplicio al De Caelo et Mundo, y obras médicas de Hipócrates y Galeno, incluidos varios tratados matemáticos de Arquímedes, dos comentarios de Eutocio; la Catoptrica de Herón y el Analema de Tolomeo. DE WULFF: Historia de la filosofia..., I, 46. Es enorme la labor de difusión de la cultura griega a los llamados "latinos", por parte de Guillermo de Moerbeke. 
ii) En segundo lugar, este dato sobre el libro XI de la Metaphysica, al que volveremos más adelante, es un punctum determinante para fechar los comentarios de Santo Tomás de Aquino $-\mathrm{y}$ partes de sus obras teológicas postremas-, así como las versiones que utilizó, según lo hace Weisheipl. Antes de esa traducción de Moerbeke, el libro XII (Lambda) se citaba como el XI (Kappa). Después de su traducción, el libro Lambda se comenzó a citar como el libro XII, lugar que actualmente ocupa en la ordenación de los libros metafísicos de Aristóteles. ${ }^{40}$

b) Clases de obras aristotélicas existentes en la Edad Media, seguin G. Lacombe

Lacombe distribuye las obras de Aristóteles a disposición de los latinos (s. XII-XIV) en seis diferentes clases. Esta distribución nos dejará ver qué obras tenía a la vista Santo Tomás, independientemente de los traductores que las habían vertido al latín, y cuáles consideraba aristotélicas aunque no lo fuesen de hecho.

1) La primera clase corresponde a las obras que aún existen en el original griego, cuya autoridad más o menos cierta, según palabras de Lacombe, se había tenido como auténtica de Aristóteles: En este grupo encontramos: Praedicamenta, Peri Hermeneias, Analytica Priora, Analytica Posteriora, De Sophisticis Elenchis, Topica; Metaphysica, Physica, De Caelo, De Generatione et Corruptione, Meteora; Historia Animalium, De Partibus Animalium, De Generatione Animalium, De Motu Animalium, De Progressu Animalium; De Anima, Parva Naturalia (De Sensu et Sensato, De Memoria et Reminiscentia, De Somno et Vigilia, De Longitudine et Brevitate Vitae, De Juventute et Senectute, De Respiratione, De Vita et Morte); Ethica Nichomachea, Ethica Eudemia, Magna Moralia, De Virtutibus et Vitiis (=De Laudabilibus Bonis); Politica, Oeconomica Vulgata, Rhetorica Vulgata, Rhetorica ad Alexandrum (Epistola ad Alexandrum); Poetica.4

40 J. WeISHEIPL: Tomás de Aquino..., p. 324.

${ }^{41}$ G. LACOMBE: Aristoteles Latinus..., Vol. I. p. 39. Tomás de Aquino cita sólo tres veces este libro de Aristóteles, y no parece haber tenido conocimiento directo de él:
Sin embargo, pongamos atención a la nota de Lacombe al citar estas fuentes: "Nostrorum temporum critici nonulli ex his operibus

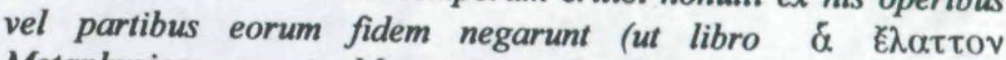
Metaphysicae, quarto Meteororum, decimo Historiae Animalium, etc.,) quod tamen nihil ad nos attinet: in nostra enim Opera aristotelicorum editione omnes huius classis libri aderunt). ${ }^{42}$ Como vemos, Lacombe no está preocupado por saber si algunos de los libros son o no aristotélicos, según algunos críticos (lo cual le correspondería a quien hiciese una edición crítica de los textos griegos), sino sólo recopilar los textos latinos que llegaron a sus manos y que eran aristotélicos, o habían pasado por aristotélicos, para los latinos medievales.

2) La segunda clase son las obras sin duda no aristotélicas, y que existen en griego bajo el nombre de Aristóteles. Entre ellas se encuentran: Problemata Vulgata, Physiognomia Vulgata, De Mundo. De Coloribus, De Mirabilibus Auditionibus, De Lineis Indivisibilibus. La mayor parte de estas obras las tradujo Bartolomeo de Messana del griego al latín. ${ }^{43}$

3) La tercera clase son las obras pseudo-aristotélicas que se tradujeron del griego al latín, y que incluso en la Grecia antigua se reconocían como obras de Aristóteles. Son las siguientes:

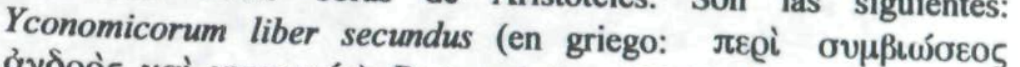

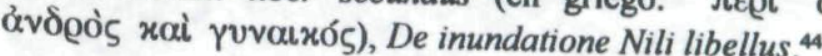

4) La cuarta clase son las obras que se decían de Aristóteles o de Teofrasto. Los latinos conocían dos libros: De Principiis (en griego: IIrei dò xw̄v, esto es, la Metaphysica de Teofrasto), y De signis

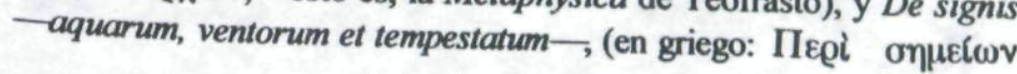

cfr. Summa Theologiae I-II, q. 32, a. 8, c.; Quaestio de Malo, q. 8, a. 3, ad 10 um; Sententia super Posteriora Analytica, Proemio. En Thome q. 8, a. 3, ad 10um. cum hypertextibus in CD-Rom, Auctore Rober. "Thomistica".

${ }^{42}$ G. LACOMBE: Aristoteles Latinus..., Vol. I. p. 39.

44 .. G. LACOMBE: Aristoteles Latinus..., Vol. I, p. 40.

Cf. G. LACOMBE: Aristoteles Latinus..., Vol. I, p. 40. 


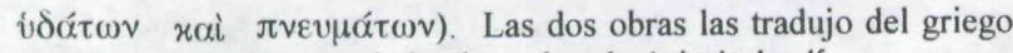
Bartolomeo de Messana bajo el nombre de Aristóteles. ${ }^{45}$

5) La quinta clase son las colecciones de Problemata aristotélicos que se leían en la baja edad media. Provienen del griego los Problemata vulgata (ver segunda clase), y, además, los Problemata Bambergensia. ${ }^{46}$.

6) Finalmente, la sexta clase contiene las obras pseudoaristotélicas traducidas desde el árabe (o del hebreo), y cuyo origen se remonta a los griegos o a los antiguos bizantinos: a) De Plantis (De Vegetabilibus et Plantis): como ya citamos, ${ }^{47}$ es un compendio de Nicolás Damasceno (siglo I a. C.) tomado a partir de la 'Botánica' de Aristóteles. b) De Causis Proprietatum Elementorum: El origen de esta obra no está determinado. Alberto Magno la comenta. c) De Congelatis (De Mineralibus): está tomado de los Meteora de Avicena, traducido del hebreo al latín por Alfredo de Sareshel. En los códices está añadido después de la traducción que hicieron Gerardo de Cremona y Enrique Aristipo al libro Meteora de Aristóteles. ${ }^{48}$

A partir de esta compilación de Lacombe, y su respectiva división en clases, hay que hacer dos consideraciones generales:

i) Las 12 obras que Santo Tomás comentó son todas originales de Aristóteles, o por lo menos se consideran así aún en la crítica contemporánea, con excepción del libro De Causis. Sin embargo, algunas de las obras que Aquino cita como existentes - aunque no comenta - no son todas aristotélicas. Tal es el caso de la obra $D e$ Mineralibus, mencionada al inicio del comentario a la Physica, o bien De Plantis, libro nombrado al inicio del comentario al De Sensu et Sensato, como diremos más adelante.

45 G. LACOMBE: Aristoteles Latinus..., Vol. I, p. 40.

${ }^{46}$ Cf. G. LACOMBE: Aristoteles Latinus..., Vol. I, p. 40.

47 Ver nota 17.

${ }^{48}$ Cf. G. LACOMBE: Aristoteles Latinus..., Vol. I, p. 41. ii) Existían varias traducciones de las obras de Aristóteles que Aquino podía confrontar a la vez, razón por la cual encontramos en sus comentarios frecuentemente las expresiones "aliam literam habet...", etc. Saber exactamente a qué versión se refería Santo Tomás en esas confrontaciones, requiere una labor exegética monumental unificada de aquellas versiones que posee la edición Aristoteles Latinus ya citada. ${ }^{49}$

\section{3) La Metaphysica de Aristóteles y las traducciones disponibles para Tomás de Aquino}

\section{a) Las versiones de la Metaphysica}

La recopilación de códices latinos de George Lacombe de las obras de Aristóteles es la fuente principal de Valentín García Yebra al presentar su traducción de la Metaphysica de Aristóteles. García Yebra sigue a Lacombe en lo referente a las diversas traducciones de la Metaphysica de las que disponemos actualmente. Hemos citado párrafos atrás dicha bibliografia (No. 2, [a]), pero conviene revisarla en particular para resaltar las diversas fuentes con las que contaba Santo Tomás. Seguimos casi al pie de la letra a Lacombe, al cual cita García Yebra, aunque de hecho lo que hace es traducirlo sin decirlo expresamente. ${ }^{50}$

a) Metaphysica Vetustissima. Descubierta y bautizada por A. Birkenmajer. Existen ocho códices en los que aparece sola o con la Metaphysica Nova. Es del siglo XII y contiene los tres primeros libros y parte del IV. Termina en la página 1007 a 32 de la edición de Bekker. Lacombe anota que el traductor de esta versión había sido Jacobo Veneto.

b) Metaphysica Medie Translationis. Descubierta y llamada así por Pelster. Existen diez códices en que aparece sola, $\mathrm{y}$ hay otros que contienen la Metaphysica Vetus más la Nova, y además, los libros

${ }^{49}$ Ver nota 9. A0 ARISTÓteles: Metaphysica..., Prólogo, p. XV y ss. Tomado de G. LACOMBE:
Aristoteles Latinus..., I, 61-66. 
XIII y XIV. Contiene, pues, los XIV libros de la Metaphysica excepto el XI. Al parecer, esta traducción es más antigua que la Metaphysica Vetus, la cual se escribió hacia 1230, según Lacombe. ${ }^{51}$ El traductor es anónimo.

c) Metaphysica Vetus. Según Lacombe, esta versión de la Metaphysica no es más que una contaminación del texto de la Vetustissima junto con el de la Media. ${ }^{52}$ Si es así, no merecería crédito la rúbrica que aparece en el Códice Burdigalensis 421, f. 257 , así como en el Matritensis Bibl. Palacii 427: Liber Aristotelis de Metaphysica Vetus translatus a Boethio de greco in latinum. La traducción fue hecha antes de 1230 (según Ross, antes de 1210) ${ }^{53}$, pues en esa fecha es utilizada por Arnoldo el Sajón.

d) Metaphysica Nova. Es una traducción del árabe, y se aparta mucho del texto griego, según cita García Yebra. Aparece hacia 1220 , y recibe el nombre de Metaphysica Nova antes de 1250 . Tiene lagunas considerables. Está dividida en 11 libros, que corresponden al libro I (cap. 5 y ss), B-I y XII (Lambda). Omite, pues, los cuatro primeros capítulos de I, todo el libro XI (Kappa) y la última parte del XII. Según Lacombe, como citamos anteriormente, probablemente la tradujo Miguel Escoto.

Al resumir este punto, García Yebra traduce las siguientes palabras de Lacombe:

Todas las traducciones latinas de la Metaphysica mencionadas hasta ahora aparecen ya en códices anteriores a 1250 , frecuentemente unidas entre sí. Esta yuxtaposición de los textos se hacía sin duda para que los maestros y las escuelas pudieran confrontarlos fácilmente y deducir de ellos con la mayor garantía posible el verdadero pensamiento del Estagirita. La práctica de la confrontación de textos se refleja

51 G. LACOMBE: Aristoteles Latinus..., I, p. 62.

52 G. LACOMBE: Aristoteles Latinus..., I, p. 63, No. 3. En "Prólogo" de Valentín Garcia Yebra, p. XVII.

53 W.D. Ross: Aristotle's Metaphysics, I, clxiv. En "Prólogo" de Valentín García Yebra, p. XVII. bien en el Comentario de Santo Tomás a los doce primeros libros, donde muchas veces se citan dos o tres versiones de un mismo pasaje..$^{54}$

e) Existe un códice Metaphysicae Fragmentum Vaticanum, cuya versión difiere de las anteriores ya citadas por ser una traducción libre del texto latino con respecto al griego. El códice sólo abarca noventa líneas, correspondientes a las 72 primeras del texto griego: I, 980a $21-981 \mathrm{~b} 13$.

f) Finalmente, la Metaphysica Nove Translationis es la versión revisada por Guillermo de Moerbeke. Guillermo tradujo el libro XI (Kappa), completando la traducción de los libros de la Metaphysica. En la obra de Lacombe nada se nos dice sobre la posible fecha de la traducción de dicho libro, dato capital para ubicar temporalmente los comentarios y redacción de algunas obras teológicas de Santo Tomás. Por su parte, Weisheipl nos refiere que la traducción del libro XI (Kappa) posiblemente tuvo lugar alrededor de 1270: " $\mathrm{La}$ datación de esa traducción es importante, porque, con la inserción del libro Kappa (libro 11), Lambda se convirtió en el libro 12; anteriormente Lambda habia sido siempre citado como el libro $11 " .55$

Podemos decir que, apreciando la variedad de textos traducidos con los que contaban los maestros de la Edad Media, en particular del siglo XIII, y en específico de la Metaphysica de Aristóteles, era posible hacer una labor de exégesis del texto a la letra. Si bien muchos maestros, como fue el caso de Tomás de Aquino, no sabían griego $\longrightarrow$ diferencia de Rogelio Bacon o Roberto Grosseteste por ejemplo $^{56}$, podían cotejar varias versiones de una misma línea o

\footnotetext{
\$4 ARISTóteles: Metaphysica..., "Prólogo" de Valentín Garcia Yebra, p. XVIII. Tomado de G. LACOMBE: Aristoteles Latinus..., I, 65.

55 J. WEISHEIPL: Tomás de Aquino..., p. 276.

56 Sobre Roberto Grosseteste (Grossum caput, Grossetête), llamado también el Linconliensis por ser Obispo de Lincoln (1235-1253), haremos solamente una breve mención de su incansable labor. Hizo traducir al latin a sus colaboradores los Testamentos de los Doce Patriarcas, las Cartas de San Ignacio de Antinquia en recensión media, las obras del Pseudo-Dionisio Areopagita, Theologia Mystica,
} 
párrafo de Aristóteles para llegar a discernir el sentido que se consideraba más adecuado respecto del pensamiento del llamado Filósofo.

\section{b) Boecio y la traducción latina de la Metaphysica}

\section{i) Boecio como traductor y comentador de la Metaphysica}

Al principio de este trabajo hemos citado un artículo sobre una posible traducción de Boecio a la Metaphysica de Aristóteles. ${ }^{57}$ Manejábamos en el mismo una hipótesis principal: "Boecio realizó una traducción y un comentario a la Metaphysica de Aristóteles, y ésta fue conocida por Tomás de Aquino"..$^{58}$ En ese texto citamos todos los pasajes donde Tomás, en sus comentarios a los libros de Aristóteles, se refiere a Boecio, apoyándose en la "littera Boethir". Interpretábamos, a partir de esas citas de Santo Tomás, que el Aquinate no solamente se basaba en una traducción literal sino también en una interpretación del mismo Severino Boecio. Tal hipótesis de trabajo también se sustenta en el hecho de que Santo Tomás cita varias versiones de la Metaphysica, e interpreta de acuerdo con lo que piensa es la verdadera intención del Estagirita.

Conviene decir, según la hipótesis de dicho artículo, basado exclusivamente en los 12 comentarios de Santo Tomás a las obras de Aristóteles, que efectivamente, hubo un apoyo en un texto latino de la Metaphysica aristotélica considerado de Boecio. Y, efectivamente,

Ierarchia Angelica, lerarchia Ecclesiastica, De Divinis Nominibus y las "Diez Cartas"; entre otros textos. Roberto por su parte tradujo los opúsculos pseudoaristotélicos: De Virtutibus et Vitiis (De Virtute o De Laudabilibus Bonis) y De Lineis Indivisibilibus, así como también el aristotélico: De Caelo et Mundo, hasta el primer tercio del libro III, cap. 1 (299a 10). Hacia 1240-43 llevó a cabo la versión grecolatina (al parecer la primera) de toda la Ethica Nichomachea. De Wulff asegura que esa versión se le atribuyó erróneamente a Guillermo de Moerbeke. Roberto incluye en su traducción a la Ethica los comentarios de Eustrato (Nicea, s. $\mathrm{XI}-\mathrm{XII}$ ) sobre los libros I y II; los de un anónimo sobre los libros II-V y VII; los de Miguel de Éfeso (s. XI) sobre los libros V, IX y X, y el de Aspasio (s. II) sobre el libro VIII. Cf. DE WulfF: Historia de la filosofia..., II, pp. 39-41.

57 J. MORÁN: “Anicio Manlio Severino...", pp. 143-166.

58 J. MORÁN: “Anicio Manlio Severino....", p. 144. como afirma De Wulf, durante el siglo XIII se dieron varias citas de la "littera Boethii" por parte de los maestros de filosofia, aunque en realidad no se sepa si esas traducciones eran o no del llamado último de los romanos. ${ }^{59}$ Las obras en las cuales el Aquinate posiblemente citaba la "littera Boethii" son la Metaphysica, caso de nuestra hipótesis, Physica y De Anima. En realidad, debe decirse que no se sabe exactamente a quién pertenecían esas traducciones que los doctores llamaban "littera Boethir" al citarla.

\section{ii) La Metaphysica Vetus}

$\mathrm{Al}$ encontrar los diversos textos (en particular de la Metaphysica) en que se basaban los maestros del siglo XIII para llevar a cabo sus comentarios, Birkenmajer y Lacombe, nombran varias versiones según las diversas épocas en que suponen que fueron elaboradas esas traducciones. Hemos citado in genere en (2 [a]) dichas traducciones que los pensadores del siglo XIII tenían a su disposición.

Ahora bien, pongamos atención al siguiente hecho. Hay un códice de la Metaphysica Vetus: Burdigalensis 421, f. 257, así como también el Matritensis Bibl. Palacii 427, titulado: "Liber Aristotelis de Metaphysica Vetus translatus a Boethio de greco in latinum". Parecería entonces que esta es la traducción de Boecio a la cual se refiere Santo Tomás en su comentario a la Metaphysica; por ello, nuestra hipótesis se podría sustentar en el título de este códice.

Sin embargo, según Lacombe, debido a que esta traducción no es sino el texto de la Metaphysica Vetustissima contaminado con el de la Metaphysica Media, no hay que hacer mucho caso a la rúbrica de Boecio como autor de esa traducción. ${ }^{60}$ Siendo ambas traducciones (Vetustissima y Media) del siglo XII aproximadamente, dicha

59 De Wulf: Historia de la Filosofia..., I, 100: “...el siglo XIII citó especialmente la littera Boethii y aun sus versiones del De Anima, de la Physica y de la Metaphysica".

${ }^{60}$ G. LACOMBE: Aristoteles Latinus..., I, 63. "Certis argumentis (quae accurate Alexander Birkenmajer mox proluturus est in Prolegomenis in Aristotelem Latinum) de quibus longius hic est dicere, hanc Met. Veterem non nisi Vetustissimae cum textu Medie Transl. contaminationem esse patet", p. 63. 
Metaphysica Vetus no podría ser de Boecio pues estaría basada en una versión cinco siglos posterior a su muerte.

Esta sería una de las objeciones más directas contra la hipótesis de que Boecio fue traductor de algunos libros de la Metaphysica aristotélica. En otros términos, Tomás de Aquino y algunos otros maestros citaban este texto en sus comentarios respectivos, llamado por la crítica moderna Metaphysica Vetus, la cual tenía como supuesto autor a Boecio, sin darse cuenta de que en realidad era una traducción tomada de versiones más recientes. Desde esa perspectiva, si cuando Tomás de Aquino cita la "littera Boethil" en realidad está citando la traducción llamada Metaphysica Vetus, nuestra hipótesis estaría basada en una suposición errónea de Tomás de Aquino y cuantos citaron esta versión.

Por otro lado, los autores de la Metaphysica Vetustissima así como de la Media no están determinados. Lacombe anota que posiblemente Jacobo Veneto es el autor de la Vetustissima, según la transcripción que hicimos parágrafos atrás, y afirma que el traductor de la Media es desconocido. La Metaphysica Vetustissima parece ser del siglo XII, y la Media también. Esta última pudo haber sido hecha en Constantinopla y es anterior a la Vetus. ${ }^{61}$

Luego de ver estos puntos debemos decir que sólo una crítica textual de las diversas litterae que Santo Tomás utiliza, en específico en su comentario a la Metaphysica, podría darnos la respuesta de tales interrogantes. ¿Es la "littera Boethii" que cita Santo Tomás en su comentario a la Metaphysica, la llamada Metaphysica Vetus? Veamos que dice De Wulff: "Birkenmajer ve en la Metaphysica vetus una contaminación de la Vetustissima con la Metaphysica Media (terminología de Pelster, constituida por los libros 1-10 y 1214, representada por el ms. Borghese 304 y parafraseada hacia 1270 por Alberto Magno)". ${ }^{2}$ Hasta aquí De Wulff afirma lo que ya hemos citado anteriormente.

${ }^{61}$ G. LACOMBE: Aristoteles Latinus..., I, 62.

${ }^{62}$ DE WULfF: Historia de la filosofia..., II, p. 37.
Sin embargo, leamos qué afirma Pelster al respecto de Birkenmajer: "Pelster niega tal contaminación y sitúa la Media después de la versión latinoárabe en 11 libros probablemente obra de Miguel Escoto- a la que los mss. intitulan Metaphysica Nova, oponiéndola a la versión parcial que era la Vetus". ${ }^{63}$ Con esta negación de Pelster notamos que quizá la Vetus no sea una 'contaminación' como afirmaba Birkenmajer, pero ciertamente no se remontaría más allá de la versión latinoárabe de Escoto, hecha siglos después de la muerte de Boecio. Nuevamente, la hipótesis principal de dicho artículo ${ }^{64}$ no resulta sostenible. ${ }^{65}$

A partir de ello cabría preguntarse de nuevo: ¿La versión Vetustissima o, incluso la Media, podrían remontarse hasta la época de Boecio? Vimos que parecen ser ambas del siglo XII porque anteriormente no se tenía noticia de ellas. El traductor de la Vetustissima que, según anotación de Lacombe, podría ser Jacobo Veneto, sólo tradujo tres libros. La Media sí tiene traducidos trece libros excepto el XI. La interrogante sobre la posibilidad de que una traducción de la Metaphysica se haya remontado hasta Boecio queda abierta, aunque, con la evidencia puesta, es muy poco plausible sostener dicha hipótesis.

Cabe asentar dos conclusiones:

a) En primer lugar, si Santo Tomás en su comentario a la Metaphysica citaba la Metaphysica Vetus, la cual tenía como probable autor a Boecio, y ésta fue hecha en el siglo XII, debe decirse que la hipótesis principal del artículo citado es equivocada. La littera Boethii citada por Santo Tomás no sería sino la Metaphysica Vetus, que en realidad se basa en la Vetustissima y la

${ }^{63}$ DE WULFF: Historia de la filosofia..., II, p. 37

${ }^{64}$ J. MorÁN: “Anicio Manlio Severino...,", p. 144.

${ }^{65}$ Para De Wulff: "La littera Boethi, como la lla

Metaphy Da littera Boethi, como la llama Santo Tomás, es idéntica a la contaphysica Vetus en los libros 1-4; difiere de la Media y aún no se encuentra su continuación" (DE WULFF: Historia de la filosofia..., II, 38). Con ello, podemos Metaphye Tomás de Aquino citaba la littera Boethii aunque en realidad citaba la traducción Vetus, por lo menos en los libros 1-4. Por ello, la hipótesis de la traducción de Boecio de la Metaphysica no resulta sostenible en esos cuatro libros. 
Media. Independientemente de las 'contaminaciones' que haya podido tener la Vetus, según hemos visto, ésta no puede ser anterior del siglo XII d.C.

b) Si Santo Tomás se refería a otra traducción, entonces cabría - muy remotamente - la posibilidad de que el autor se remontara hasta Boecio mismo.

\section{iii) El libro XI de la Metaphysica: traducción tardía}

Es capital la importancia de la traducción del libro XI (Kappa) de la Metaphysica puesto que sirve para saber de modo aproximado las fechas de composición de diversos comentarios de Santo Tomás:

Hay cuatro criterios generales utilizados por los especialistas para datarlos (los comentarios), a falta de una referencia concreta en los mismos manuscritos. Primero, el criterio Lambda de la Metaphysica, es decir, la fecha en que el libro $\mathrm{K}$ (XI) fue nuevamente traducido del griego por Moerbeke e insertado en la Metaphysica como el libro XI, por lo que Lambda, antiguamente el libro XI, se convirtió en el libro XII. En segundo lugar, hay ciertas fuentes utilizadas por Tomás, especialmente griegas, que pueden ser fechadas directamente. Tercero, la versión latina de Aristóteles utilizada por Tomás. Finalmente, tenemos el desarrollo de la doctrina: este es el criterio más difícil de todos, pero de gran importancia. ${ }^{66}$

Según afirma Weisheipl, quien es muy congruente con su sentencia, ${ }^{67}$ el primero de los criterios para fechar los comentarios es la traducción del libro XI de la Metaphysica. Tal traducción pudo

66 J. WeISHELPL: Tomás de Aquino..., p. 325.

${ }^{67}$ Ver por ejemplo, el Suplemento del libro de Weisheipl citado, en donde hace un recuento de las obras de Santo Tomás, originales y espúreas. Las fechas de la composición de algunas partes del comentario a la Metaphysica misma dependen del dato de esa traducción de Moerbeke. J. WeISHEIPL: Tomás de Aquino..., pp. 428 ss. haber sido hecha en $1270 .{ }^{68}$ Aquí cabe otra interrogante: ¿por qué no fue traducido el libro XI (Kappa) de la Metaphysica de Aristóteles sino hasta entrado el siglo XIII? ¿Era absolutamente desconocido dicho libro? Hacemos esta última pregunta porque ciertamente cuando Santo Tomás cita el libro XII como el XI —no teniendo aún la traducción del libro $\mathrm{XI}-$, no hace ninguna mención de la falta de traducción, como sí lo hace en el caso de los libros XIII y XIV de la misma Metaphysica. ${ }^{69}$ Ello indica que no había noticia de la ausencia de tal libro aristotélico.

Existe una cuestión antecedente a Tomás de Aquino de modo directo que nos puede dar una pista. Alberto Magno comentó trece libros de la Metaphysica de Aristóteles. En efecto, Alberto comenta XIII libros de la Metaphysica, y llama libro XI al que nosotros conocemos como el XII (Lambda), y así sucesivamente. ${ }^{70}$ Los libros XII y XIII son para Alberto Magno los libros XIII y XIV respectivamente para nosotros. Al hacer, pues, su comentario a la Metaphysica, Alberto no menciona la existencia del libro XI (Kappa).

Por eso repetimos la pregunta, si el libro XI de la Metaphysica sólo fue conocido por medio de la traducción de Moerbeke a partir del griego, sin antecedente latino ninguno, ¿cómo llegó a manos del mismo Moerbeke?," ¿de dónde surgió dicha versión griega para

${ }^{68}$ Cf. J. WeISHeIPL: Tomás de Aquino..., p. 276.

${ }^{69}$ Ver nota 73.

70 B. Alberti Magni: Ratisbonensis Episcopi, Ordinis Praedicatorum, Opera Omnia, Cura ac labore Augusti Borgnet, Vol. VI, "Metaphysicorum lib. XIIr", Parisis, 1890. Analizando la temática del libro que Alberto llama XI, se nota que, en realidad, es el que nosotros conocemos como XII (Lambda)

${ }^{71}$ El Prof. Enrique Alarcón (Universidad de Navarra) nos respondió directamente al respecto: "Los primeros documentos históricos sobre Guillermo de Moerbeke son us traducion son su presencia alli quizá estriba en 1260 . La razón de Hazebrouck a en que el sorío de Moerbeque, cerca de familia de le al Norte de Francia, pertenecía entonces a la Casa de Saint Omer, enilia de las más poderosas en el señorío de Tebas. Obviamente, nada hay de raro en la presencia de mss. griegos en el Peloponeso. En cuanto a los mss. griegos pontificios, suelen tener la anotación Aud.: yo lo interpreto Audomari, a saber, de la casa de Omer, a cuyo servicio estuvo Moerbeke en Tebas". Carta: 22-07-2002. 
poder ser traducida? ${ }^{72}$ ¿Qué versión griega poseía Moerbeke en Grecia que fuese completa, y, más aún, por qué esa versión no llegó a los latinos para ser traducida dos siglos antes? No podemos ir más adelante en estas preguntas porque sería como intentar llenar un barril sin fondo.

A manera de epílogo hay que preguntarnos de nuevo: ¿por qué Santo Tomás en un texto tan cercano a su muerte como De Unitate Intellectus, ca. 1271, dice que no ha llegado hasta él la traducción de los últimos libros (XIII-XIV) de la Metaphysica de Aristóteles? ${ }^{73} \mathrm{Si}$ es que la Metaphysica Media se encontraba a la mano de los maestros en el siglo XIII, ¿por qué Tomás hace esa aseveración? Alberto Magno comenta incluso los libros XIII y XIV (para él XII y XIII) de la Metaphysica, razón por la cual parecería que sí existían esas traducciones. Sin embargo, Santo Tomás no las tuvo al

72 Sobre este problema afirma De Wulff: "En cuanto a la procedencia de los mss 2 Sobre cste problema afirma De Wu traducción griegos, utilizados por Guillermo (Moerbeke) en su empresa de traducción revisión, los vio durante su permanencia en el principado de Acaya, en el ducado de Atenas y en Nicea. capital del nuevo imperio griego. Otros los encontró en la curi Atenas y en Nicea, capital dentre los mss. donados al papa Clemente IV por Carlos I de romana, particularmente entre los mss. don batalla de Benevento. Anjou y separados del botín obtenido (febrero de 1266 ) en la batalla de Benevento. donde sucumbió Manfredo. Varios de estos manuscritos griegos figuran en lo inventarios del tesoro pontificio, formados en 1295 y 1311 ". DE WULFF: Historia de la filosofia. II. p. 47. De Wulff tampoco nos aclara mayor cosa sobre este la filosofia.... 11. p. 47. De Wuntar: ¿cómo llegaron al principado de Acaya esos particular. Podemos volver a preguntar: ¿como llegaron al principado de Acaya esos manuscritos?, y aun, ¿por qué no habian llegado antes a los latinos esos mismos

73 ....illam partem metaphysicae non habemus, quam fecit (Aristoteles) de substantiis separatis" ("aquella parte de la Metaphysica no la tenemos, la cual hizo [Aristóteles] sobre las sustancias separadas"). Cf. Sancti Thomae AQuinatis: De Unitate Intellectus. Cap. 5, Lin. 393. En Thomae Aquinatis Opera Omnia cum Unitate Intellectus, CD-Rom, Auctore Roberto Busa S.J. Ed. 1996, Secunda. hyperlextibus in Co "Thomistica". Según la redaccion de Aquino, puede ser que. a) la traducción de esos dos libros (XIII-XIV) no la tenía en sus manos en ese momento, puesto que los códices debian ser escasos; b) esa traducción no la tenían en sus manos los maestros de Paris; c) sólo se conocía la existencia de tales libros, pero no se conocia su de Paris, c) solema con (c) es que Alberto tematica, d) esta linea del texto sea apocina. El problos libros I-XIV, excepto e Magno comentó XIII libros de la Metaphysica, es decir, los libros l-XIV, excepto e XII, según dijimos. De ahí nuestra pregunta: si el comentario de Tomás de Aquinu parece ser posterior al de Alberto Magno ¿por qué no tenía a la mano esos dos libros? momento de hacer su comentario por razones desconocidas para nosotros.

\section{4) Obras aristotélicas citadas en el comentario de Tomás de Aquino al libro De Sensu et Sensato de Aristóteles}

Luego de vistas en general las obras de Aristóteles que los "latinos" del siglo XIII tenían a su disposición, incluso algunas pseudo-aristotélicas, así como notas respectivas a la Metaphysica de Aristóteles, debemos terminar estas aproximaciones a tales temas haciendo un recuento de los textos aristotélicos que Santo Tomás ordena en el prooemium al libro aristotélico De Sensu et Sensato. ${ }^{74}$ Es ahí donde más obras de Aristóteles son citadas conjuntamente, siendo jerarquizadas según diversos criterios cognoscitivos, como veremos.

Hemos visto en el inciso (2 (b)) que G. Lacombe clasifica en seis clases las obras de Aristóteles traducidas al latin. La primera clase se refiere a los libros aristotélicos originales. Santo Tomás de Aquino cita por lo menos en dos ocasiones algunas obras que no son aristotélicas, aunque en el siglo XIII pasaban como aristotélicas. Tales son De Mineralibus, De Plantis y, añadimos ahora, De Nutrimento et Nutribile.

Tomás, contextualizando el 'lugar' del libro De Sensu et Sensato en la filosofia natural, hace un recuento de obras aristotélicas naturales utilizando tres criterios para la posible ordenación de esos libros. Veamos qué obras cita Aquino en su ordenación, como aparecen en la versión Marietti. A la vez, citaremos los nombres de estos textos según ediciones modernas, tal como se encuentran en la edición Didot y según las denominaciones de Lacombe.

${ }^{74}$ Sancti Thomae AQuinatis: In Aristotelis libros de Sensu et Sensato. de Memoria et Reminiscentia Commentarium, cura et studio P. Fr. Raymundi M. Spiazzi. O.P. Torino: Marietti, 1949, Prooemium, p. 4. Citaremos esta obra como: In de Sensu et
Sensato. 
a) La primera ordenación de las obras naturales de Aristóteles sigue el criterio de la separabilidad de la materia. ${ }^{75}$ Según dicho criterio, se estudia primero lo más universal, lo más separado de la materia, y posteriormente lo más particular. Aplicado a la ordenación y jerarquía de los libros tenemos el siguiente resultado. Los textos De Anima, De Sensu et Sensato y el 'de animalibus et plantis' son citados por Santo Tomás. Sin embargo, notemos que no hay ninguna obra aristotélica llamada De Animalibus et Plantis, tal como se encuentra redactado el texto latino de Aquino.

En la publicación de Marietti que citamos ahora, se afirma que probablemente Santo Tomás se refiere a: De Animalibus Historia, De Partibus Animalium y De Generatione Animalium, según los nombres que proporciona la Edición Didot. ${ }^{76}$ La obra De Plantis sí aparece en los códices de Lacombe, aunque es pseudo-aristotélica, según vimos en su momento.

b) La segunda ordenación de las obras naturales sigue el criterio de los grados de vivientes. ${ }^{77}$ Según este orden, suponiendo el libro De Anima, primero se estudia el viviente en cuanto tal.

1) Primero menciona Santo Tomás: De Morte et Vita, en donde se determina 'de respiratione et expiratione'. A su vez, hace mención al De Iuventute et Senectute, asi como Causis Longitudinis y Brevitate Vitae; finalmente De Sanitate et Aegritudine, y De Nutrimento et Nutribile.

i) Vemos en esta ordenación que De Morte et Vita, Santo Tomás lo considera un libro y, según su redacción latina: "ahí se determina sobre la respiración y la expiración". La edición Marietti anota la partícula: 'determinat de respiratione et expiratione', como si fuese el libro 'De respiratione et expiratione'. Según vimos en la primera clasificación de las obras aristotélicas $(2,[\mathrm{~b}]), D e$

\footnotetext{
${ }^{75}$ S.T. AQuinatis: In de Sensu et Sensato, Prooemium.

${ }^{76}$ Aristotelis Opera Omnia: Index Nominum et Rerum...

${ }^{77}$ S.T. AQUinatis: In de Sensu et Sensato, Prooemium.
}

Respiratione está clasificada entre las obras aristotélicas latinas de la primera clase, según nomenclatura de Lacombe.

ii) La obra Ilamada en el proemio al De Sensu, según Aquino: "Causis Longitudinis" y "Brevitate Vitae", como separando el nombre en Marietti, es, sin embargo, llamada, tanto en la numeración de Lacombe como en la edición Didot: De Longitudine et Brevitate Vitae. Por otro lado, en la numeración de Lacombe no aparecen: De Nutrimento et Nutribile ni De Sanitate et Aegritudine. ${ }^{78}$ A su vez, esta última obra según la edición Marietti, tampoco aparece en la edición Didot. ${ }^{79}$ La obra De Nutrimento et Nutribile, de la cual, como menciona el Aquinate 'no nos llegaron dos libros' (qui duo libri apud nos nondum habentur), ${ }^{80}$ si es comentada por Alberto Magno. ${ }^{81}$

2) Segundo, Aquino se refiere al De Causa Motus Animalium y De Progressu Animalium. Según la Edición Marietti, el primer libro se encuentra en Didot como De Animalium Incessu. Según Lacombe hay una obra llamada De Motu Animalium, así como también existe De Progressu Animalium, las cuales podrian ser esa que menciona individualmente Aquino. El libro llamado De Progressu Animalium, según Marietti, en Didot tiene el título De Partibus Animalium. En Lacombe encontramos De Progressu Animalium, la cual podría ser dicha obra. ${ }^{82}$

3) Tercero, Tomás menciona De Sensu et Sensato, De Memoria et Reminiscentia y De Somno et Vigilia. Obras que, como

\footnotetext{
${ }^{78}$ G. LACOMBE: Aristoteles Latinus..., I, 59-60.

79 S.T. Aquinatis: In de Sensu et Sensato, Prooemium.

${ }^{80}$ S.T. AQuinatis: In de Sensu et Sensato, Prooemium.

${ }^{81}$ D. Alberti MagnI: Liber de Nutrimento et Nutribile, Tractatus I: De nutrimento et nutrito per se; II: De nutrimento et nutrito, en 'Parva Naturalia'. D. ALBERTI MAGNI: Ratisbonensis Episcopi, Ordinis Praedicatorum. Operum B. Alberti Magni quae vulgo Parva Naturalia nuncupantur et in volumine nono continentur, Opera Omnia, Vol. 9, pp. 323-341. En realidad, se trata de una obra menor.

${ }^{82}$ G. LAcomBE: Aristoteles Latinus..., I, 39.
} 
hemos visto anteriormente, Lacombe pone en la primera clase de textos aristotélicos.

c) La tercera ordenación de los libros naturales de Aristóteles, dice Aquino, se da según la semejanza y la desemejanza. ${ }^{83}$ Santo Tomás cita De Anima y De Sensu et Sensato. Como hemos citado, también se encuentran dentro del primer grupo que Lacombe acepta como textos aristotélicos genuinos.

En resumen, hay obras pseudo-aristotélicas que Tomás de Aquino cita en su proemio al De Sensu et Sensato, a saber: De Nutrimento et Nutribile y De Sanitate et Aegritudine. A su vez, hay obras que tenian nombres diversos a los actuales. Tales son las obras sobre los animales: De Causa Motus Animalium, conocida hoy como De Animalium Incessu; De Progressu Animalium citada como De Partibus Animalium. La causa de ello la encontramos en la traducción de Miguel Escoto de los libros naturales de Aristóteles, en el siglo XIII. Se le atribuye a Escoto dicha traducción y una ordenación en XIX libros sobre los animales. ${ }^{84}$

Relacionado con ello, Lacombe cita el códice Vat. lat. 2093 (f. 1) en cuyo margen se encuentra la siguiente anotación: "Nova translatio libri De Animalibus continet libros 19 et habet tres partes principales que vocantur De Historiis Animalium et continet $X$ libros primos; secunda que vocatur De Partibus Animalium et contine libros sequentes, scilicet 11, 12, 13, 14; tertia que vocatur De Generatione Animalium continet 5 sequentes post 14, scilicet 15, 16, $17,18,19^{\prime \prime} .85$

Esta sería la explicación de por qué los latinos citaban los libros de los animales como el "XV De Animalibus", etc., ya que el corpus de obras naturales aristotélicas se tomaba como este todo mencionado por Lacombe. Asi, Historia Animalium estaria constituida de X

${ }^{83}$ S.T. AQuinatis: In de Sensu et Sensato, Prooemium.

84 G. LACOMBE: Aristoteles Latinus..., I, 80. “Omnes libros De Animalibus quos Arabes cognoverunt vertit in latinum Michael Scolus, eiusque translationi titulus 'De Animalibus libri XIX' saepe datus est apud latinos".

${ }^{85}$ G. LACOMBE: Aristoteles Latinus..., I, 82 libros (1-10); De Partibus Animalium, de IV (11-14); y De Generatione Animalium, de V (15-19). Los otros dos añadidos serian entonces De Motu Animalium y De Progressu Animalium. Así se tiene el corpus de XXI obras referidas a los animales. Aquino, como hemos visto, cita dos de estos libros como parte de las obras del viviente en el proemio al De Sensu et Sensato.

\section{5) Epilogo}

a) De las obras aristotélicas que tenían los "latinos", Santo Tomás poseia varias versiones a la mano (de la Metaphysica hasta tres), y comentó 12 de ellas. Incluso comentó una obra pseudo-aristotélica: Liber de Causis, aunque en su momento se dio cuenta de que ésta no era original de Aristóteles.

b) Comentando la Metaphysica de Aristóteles, Tomás de Aquino se basó tanto en versiones antiguas como en revisiones de G. Moerbeke, en específico la nueva revisión de la Metaphysica.

c) Respecto de la Metaphysica hace falta un aparato crítico del texto latino que utilizó Santo Tomás para determinar en cada pasaje específico- las versiones que utilizó en diversas partes de su comentario.

c) Aquino en sus comentarios hace mención de obras que indudablemente eran pseudo-aristotélicas, tales como De Plantis, De Mineralibus, De Nutrimento et Nutribile, aunque no hay comentario
sobre ellas.

\section{6) Apéndice}

Tomás de Aquino comentó doce obras de Aristóteles, según hemos visto en nuestro trabajo. Comentó además el pseudo-aristotélico De causis. En nueve de esos trece comentarios, Aquino se refiere por lo menos una vez a otra versión del texto aristotélico en latín que tenía en sus manos. Citaremos a continuación los lugares específicos en 
esos nueve comentarios de Tomás de Aquino, en donde se encuentra la expresión "secundum aliam litteram". ${ }^{86}$

i) Sententia super Peri Hermeneias: 1 cita

1. L. II, Lect. II, No. 15

ii) Sententia super Physicam: 6 citas

1. L. I, Lect. IX, No.16

2. L. II, Lect. V, No.10

3. L. III, Lect. X, No. 3

4. L. V, Lect. X, No.11

5. L. VII, Lect. III, No. 9

6. L. VII, Lect. III, No. 9

iii) Sententia super De Caelo et Mundo: 3 citas

1. L. I, Lect. XV, No. 1

2. L. II, Lect. XVII, No. 2

3. L II, Lect. XVIII, No. 3

iv) Sententia super libros De Generatione et Corruptone: 1 cita

1. L. I, Lect. 5 , No. 6

v) Sententia super De Anima: 2 citas

1. L. II, Lect. XXIV, No. 9

2. L. II, Lect. XXIV, No. 12

vi) Sententia de Sensu et Sensato: 2 citas

1. Lect. III, No. 11

${ }^{86}$ Para esta labor, nos hemos servido del CD-Rom que contiene la obra completa de Santo Tomás, ya citado en este trabajo: Thomae Aquinatis Opera Omnia cum hypertextibus in CD-Rom, Auctore Roberto Busa S.J. Ed., 1996, Secunda "Thomistica". Anotamos el libro, la lección y el número del pasaje correspondiente.
2. Lect. XI, No. 8

vii) Sententia super de Memoria et Reminiscentia: 1 cita

1. Lect. IV, No. 8

viii) Sententia libri Ethicorum: 3 citas

1. L. VI, Lect. VII, No. 20

2. L. IX, Lect. XIII, 13, No. 18

3. L. IX, Lect. XIV, No. 2

ix) Sententia super Metaphysicam: 43 citas

1. L. I, Lect. I, No. 23

2. L. I, Lect. I, No. 23

3. L. I, Lect. I, No. 31

4. L. I, Lect. I, No. 33

5. L. I, Lect. IV, No. 4

6. L. I, Lect. V, No. 7

7. L. I, Lect. VI, No. 8

8. L. I, Lect. XIV, No. 2

9. L. III, Lect. X, No. 9

10. L. III, Lect. XIV, No. 2

11. L. IV, Lect. V, No. 7

12. L. IV, Lect. V, No. 15

13. L. IV, Lect. VII, No. 6

14. L. IV, Lect. VIII, No. 13

15. L. IV, Lect. IX, No. I

16. L. IV, Lect.IX, No. 2

17. L. IV, Lect. XII, No. 4

18. L. IV, Lect. XII, No. 5

19. L. IV, Lect. XII, No. 9

20. L. IV, Lect. XII, No. 13

21. L. IV, Lect. XIII, No. 2

22. L. IV, Lect. XIV, No. 3

23. L. IV, Lect. XV, No. 2

24. L. IV, Lect. XVI, No. 9 
25. L. V, Lect. I, No. 3

26. L. V, Lect. II, No. 12

27. L. V, Lect. V, No. 1

28. L. V, Lect. V, No. 4

29. L. V, Lect. V, No. 6

30. L. V, Lect. V, No. 7

31. L. V, Lect. V, No. 10

32. L. V, Lect. VI, No. 3

33. L. V, Lect. VII, No. 8

34. L. V, Lect. XIII, No. 9

35. L. V, Lect. XVI, No. 6

36. L. V, Lect. XX, No. 11

37. L. V, Lect. XX, No. 11

38. L. V, Lect. XXI, No. 19

39. L. V, Lect. VI, No. 13

40. L. VII, Lect. VI, No. 33

41. L. VII, Lect. XI, No. 33

42. L. VII, Lect. XVI, No. 17

43. L. VIII, Lect. I, No. 5

\section{Hombre, mundo y lenguaje en la ontología hermenéutica de Hans-Georg Gadamer}

Francisco Fernández Labastida Pontificia Università della Santa Croce

In his masterwork, Truth and Method, Gadamer seeks to resolve the fracture between being and truth produced in Modernity by the primacy of self-consciousness and scientism. Gadamer finds in language (logos) the ultimate ground for the correspondence of finite human intellect and being, without being forced to return to the classical metaphysical assertion that held God as the foundation of the adaequatio rei et intellectus. For Gadamer, in language man and world are inseparably intertwined. As a consequence, within logos every ontological difference disappears, dissolving the being of man into the infinite historical becoming of the real protagonist: language.

\section{De la interpretación de textos a la hermenéutica filosófica}

En su acepción tradicional, el término "hermenéutica" indica cualquier actividad de interpretación, desde la traducción de una lengua a otra a la explicación e interpretación de las oscuridades o dificultades de cualquier texto, ya sea éste sagrado o profano. Sin embargo, esta noción ha sufrido cambios profundos en los dos últimos siglos. A partir del siglo diecinueve, su campo semántico se ha ido ampliando gradualmente, gracias a las reflexiones acerca del fundamento de la interpretación hechas por Friedrich Schleiermacher. Para la conciencia crítica de este teólogo romántico, no sólo los textos o discursos requieren interpretación, sino toda expresión histórica del hombre - ya sea ésta religiosa, artística o Agradezco a los Prof. Francesco Russo y Stephen L. Brock sus sugerencias y
comentarios. 
Copyright of Tópicos. Revista de Filosofía is the property of Universidad Panamericana and its content may not be copied or emailed to multiple sites or posted to a listserv without the copyright holder's express written permission. However, users may print, download, or email articles for individual use. 